\title{
Quantum steps to better sequencing
}

\author{
Nanopores and electron tunnelling offer a potentially revolutionary approach to DNA sequencing.
}

The first draft sequences of the human genome were completed around ten years ago $^{1,2}$, opening up the possibility of medical treatments being tailored to individuals on the basis of their genetic make-up. It took 13 years and cost an estimated $\$ 3$ billion to complete the first genome, but sequencing times and costs have fallen significantly since then. The 1000 Genomes Project, for example, has recently reported sequencing the entire genomes of 179 individuals and characterizing all the protein-coding sequences of another 697 people $^{3}$. Yet DNA sequencing remains too slow and expensive (around \$100,000 per human genome) to be used routinely, so there is a race to develop new technologies that are capable of sequencing genomes for $\$ 1,000$ or less - a cost that would allow sequencing to become an integral part of an individual's medical care ${ }^{4}$.

A single strand of DNA is comprised of a sequence of bases - adenine, cytosine, guanine and thymine - that are attached to a backbone made of sugar-phosphate units. Two antiparallel strands combine to form a double helix, with the strands held together by hydrogen bonds between complementary bases: adenine always pairs with thymine, and cytosine with guanine. A chief part of the challenge in DNA sequencing is that the human genome contains an estimated 3 billion of these base pairs.

The standard method for sequencing DNA, developed by Fred Sanger in the 1970s (ref. 5), is to isolate a strand of DNA, replicate it and then cut it into numerous very short strands. These short stretches of DNA are then used as templates to create an assortment of complementary strands of varying length that always begin at the same base on the template and are terminated with a modified DNA base tagged with a fluorescent marker. These complementary strands can be separated using capillary electrophoresis - in which the DNA is driven through a gel by an electric field and detected by exciting the fluorescent tag with a laser. The short DNA strands have overlapping sequences and can be reassembled to reveal the original sequence.

The Sanger method has undergone numerous refinements over the years but it remains expensive and has a number of inherent limitations. In particular, the longest strand of DNA that can be effectively read with electrophoresis is restricted to around 1,000 base pairs. Of all the alternative technologies being explored, DNA sequencing with nanopores is arguably the most revolutionary ${ }^{6}$.

The nanopore approach to sequencing involves threading a single strand of DNA through a synthetic or natural pore that is around two nanometres in diameter. The DNA is driven through the pore under an applied potential and modulations in the ionic current passing through the pore are used to identify the molecule. The technique could potentially directly read the bases of large DNA molecules without any chemical modification of the DNA - and thus provide a fast, low-cost sequencing method. Considerable progress has been achieved in nanopore sequencing, but significant issues still need to be resolved including obtaining single-base resolution.

Ion-current measurements are, however, not the only readout mechanism being considered for nanopore sequencing - the quantum-mechanical phenomenon of electron tunnelling has been proposed as an alternative. The phenomenon involves an electron of a given energy tunnelling through a potential barrier that would repel a classical particle with the same energy, and it allows a current to flow between two electrically biased electrodes that are separated by a small gap. The tunnelling current is extremely sensitive to the distance between the two electrodes and, moreover, to atoms and molecules placed between the electrodes.

The idea that electron tunnelling across a DNA molecule could be used for sequencing was first proposed in 2005 by James Lee and Thomas Thundat in a patent application ${ }^{7}$ and, independently, by Michael Zwolak and Massimiliano Di Ventra ${ }^{8}$. It was also recently shown experimentally that the approach can distinguish individual nucleotides (a base joined to a sugar and one or more phosphate groups) and individual nucleosides (a base joined to a sugar) ${ }^{9}$.

On page 868 , Stuart Lindsay and colleagues now report that the single bases in short DNA molecules can be identified with electron tunnelling. The molecules are passed through a nanoscale tunnelling gap formed between a gold surface and the tip of a scanning tunnelling microscope, both of which have benzamide groups $\left(\mathrm{C}_{6} \mathrm{H}_{5} \mathrm{CONH}_{2}\right)$ attached. These chemical groups can bind, by means of hydrogen bonds, to the different bases as the DNA molecules pass through the tunnelling junction. And Lindsay and co- workers find that the current signals obtained from the bases in the short strands are very similar to those of the isolated nucleotides. As a result, tunnelling can be used to distinguish all of the different bases in a DNA sequence.

A variety of fundamental and practical issues need to be addressed before the combination of nanopores and tunnelling emerges as a viable sequencing technology. For example, at present the DNA randomly passes through the tunnelling junction created between the gold surface and the scanning tunnelling microscope tip. To be effective, the DNA would instead need to be forced to pass one base at a time through a tunnel junction at a controlled speed (see page 828). Encouragingly, conductive nanopores have recently been reported that would perhaps allow electron tunnelling measurements to be made in devices capable of such control. In particular, earlier this year it was shown DNA molecules could translocate through single-walled carbon nanotubes ${ }^{10}$. Furthermore, graphene membranes have very recently been used as nanopore sensors ${ }^{11}$, and the use of graphene nanoelectrodes for DNA sequencing has already been theoretically considered ${ }^{12}$.

At the same time, important advances continue to be made with more traditional nanopore devices. On page 874, Cees Dekker and colleagues report hybrid nanopores in which an $\alpha$-haemolysin protein pore is inserted into a silicon nitride nanopore. This approach combines the advantageous properties of biological pores, such as an atomically precise structure, with those of synthetic nanopores, such as durability. Moreover, like base reading with electron tunnelling, hybrid nanopores provide another intriguing future direction for DNA sequencing with nanopores.

\footnotetext{
References

1. International Human Genome Sequencing Consortium Nature 409, 860-921 (2001).

2. Venter, J. C. et al. Science 291, 1304-1351 (2001).

3. The 1000 Genomes Project Consortium Nature 467, 1061-1073 (2010).

4. http://www.genome.gov/12513210

5. Sanger, F., Nicklen, S. \& Coulson, A. R. Proc. Natl Acad. Sci. USA 74, 5463-5467 (1977).

6. Branton, D. et al. Nature Biotechnol. 26, 1146-1153 (2008).

7. Lee, J. W. \& Thundat, T. US patent 6,905,586 (2005).

8. Zwolak, M. \& Di Ventra, M. Nano Lett. 5, 421-424 (2005).

9. Thundat, T. Nature Nanotech. 5, 246-247 (2010).

10. Liu, H. et al. Science 327, 64-67 (2010).

11. Siwy, Z. S. \& Davenport, M. Nature Nanotech. 5, 697-698 (2010).

12. Postma, H. W. C. Nano Lett. 10, 420-425 (2010).
} 